\title{
Avaliação Preliminar de Ambiente Online Baseado em Jogos Digitais: Um Estudo de Caso na Aprendizagem de Algoritmos
}

\author{
Daniel Antonio Karling ${ }^{1}$, Claudia Brandelero Rizzi ${ }^{1}$, Rogério Luis Rizzi ${ }^{1}$ \\ ${ }^{1}$ Universidade Estadual do Oeste do Paraná (UNIOESTE) \\ Cascavel - PR - Brasil \\ \{daniel.karling, claudia.rizzi, rogerio.rizzi\}@unioeste.br
}

\begin{abstract}
Resumo. Estudos apontam que a falta de motivação e de conhecimentos prévios por parte dos alunos, e o emprego de material didático inadequado pelo professor, contribuem para desistências e reprovações em cursos na área da Computação. Buscando avançar nas discussões pertinentes e contribuir para mitigar esses problemas, este trabalho relata uma avaliação preliminar de um ambiente online de aprendizagem de algoritmos baseado em jogos digitais, desenvolvido e concebido a partir de uma sequência didática fundamentada da Teoria da Aprendizagem Significativa. Os resultados mostram que o ambiente possui potencial de aprendizagem, com nota de 81 entre 0 e 100, e de motivação em continuar aprendendo algoritmos, com média de 3,1 na escala likert (0-5).
\end{abstract}

\begin{abstract}
Studies point out that the lack of motivation and previous knowledge for the students, and the use of inappropriate teaching material by the teacher, contribute to dropouts and failures in courses of Computing. This work reports a preliminary assessment of an online environment for learning algorithms based on digital games, developed from a didactic sequence based on the Meaningful Learning Theory, in order to advance the pertinent discussions and aiming to contribute to mitigate these problems. The results show that the environment has learning potential, with a score of 81 between 0 and 100, and motivation potential to continue learning algorithms, with an average of 3.1 on the likert scale (0-5).
\end{abstract}

Palavras-chave: Ambiente de aprendizagem de algoritmos. Jogos digitais. Aprendizagem Significativa.

\section{Introdução}

A sociedade apresenta-se fortemente impactada pelas Tecnologias Digitais da Informação e Comunicação (TDIC) e sua disponibilização e acesso têm colocado desafios e oportunidades também ao Setor Educacional. A necessidade de ferramentas digitais e seu uso combinado de metodologias adequadas evidenciaram-se ainda mais com o cenário imposto pela COVID-19 e a educação remota emergencial [Hodges et al. 2020]. Esse contexto pode ter acentuado ainda mais a problemática da formação de graduandos e técnicos em Computação, pois estudos já apontavam dificuldades decorrentes de diversos fatores, entre eles a falta de motivação dos alunos [Petrovskaya 2019], a necessidade de operar cognitivamente de modo abstrato, o pouco conhecimento de conteúdos relevantes à área por parte dos ingressantes, além de emprego de materiais e condução didática inapropriados por parte dos professores [Giraffa and da Costa Mora 2016], entre outras questões. 
Alguns desses fatores estão relacionados com ideias centrais da Teoria da Aprendizagem Significativa, que é fundamentada na concepção de que o conhecimento é construído ao longo de um processo interativo entre a nova informação e as estruturas cognitivas préexistentes do aprendiz. Nesse sentido, a Teoria considera que são três os aspectos principais que uma metodologia de ensino deve se embasar, sendo eles a existência de conhecimentos prévios relevantes, a predisposição a aprender por parte dos educandos, e o emprego de materiais potencialmente significativos pelos educadores [Ausubel 2000]. No sentido da predisposição a aprender, há evidências de que a aprendizagem baseada em jogos digitais, oportunizada pelas TDIC, apresenta potencial importante devido a sua característica lúdica [Prensky 2001, Annetta et al. 2009, Petrovskaya 2019]. A literatura mostra resultados positivos e espaço para melhorias, em especial quanto a conduzir alunos sem nenhum conhecimento em programação e a explicitar conteúdos teóricos durante a realização de atividades práticas [Shahid et al. 2019, Miljanovic and Bradbury 2018].

Em resposta a estas oportunidades de melhorias, um dos objetivos de um projeto maior ao qual os autores do presente trabalho estão vinculados, é desenvolver e disponibilizar um ambiente online de aprendizagem baseado em jogos digitais, como material potencialmente significativo, visando contribuir positivamente na predisposição do aluno a aprender e em uma tentativa de promover a Aprendizagem Significativa em conteúdos iniciais de Algoritmos. Um estudo de caso foi realizado com graduandos em Ciência da Computação de uma universidade pública, matriculados na disciplina de Algoritmos, com o propósito de avaliar o ambiente online desenvolvido. Este, materializa os contributos das concepções teóricas adotadas e visa o estímulo à motivação e melhor compreensão dos conteúdos de algoritmos por parte dos alunos. O presente trabalho objetiva apresentar os resultados de uma avaliação preliminar do ambiente, que indicaram seu alto potencial educacional e apontaram aspectos que podem ser melhorados. As seções que se seguem apresentam: a fundamentação teórica e trabalhos relacionados; metodologia de pesquisa empregada; os resultados; e considerações finais e trabalhos em andamento e futuros.

\section{Fundamentação Teórica}

\subsection{Teoria da Aprendizagem Significativa}

Segundo a Teoria da Aprendizagem Significativa (TAS), a estrutura cognitiva organizase de maneira hierárquica, situando os conceitos mais gerais e inclusivos no topo, viabilizando a assimilação e organização dos mais específicos que a eles se relacionam. Para a aprendizagem, os conhecimentos prévios relevantes do aluno - subsunçores - devem estar relacionados de maneira não arbitrária e substancial, aos novos conhecimentos com os quais ele tem vínculo, sendo modificados pela aquisição de novos significados; uma síntese dessas discussões estão em [Moreira 2010]). É fundamental que o aprendiz tenha acesso a materiais instrucionais potencialmente significativos e que apresente predisposição a aprender, opção voluntária e consciente. Um dos grandes efeitos positivos da motivação é o aumento da atenção, além de que a consciência de uma aprendizagem bem sucedida estimula o aluno a continuar aprendendo [Ausubel 2000].

Para o desenvolvimento do ambiente online procurou-se relacionar o conteúdo teórico com conceitos simples e do cotidiano, de modo que o aluno pudesse resolver desafios no decorrer do enredo dos jogos aplicando conhecimentos no âmbito da temática de algoritmos. A ordem em que os conceitos são abordados foi estruturada de maneira 
lógica e hierárquica, partindo dos aspectos mais gerais, estruturantes, e avançando para para os níveis mais específicos, consistente com a Teoria.

\subsection{Aprendizagem baseada em jogos digitais}

No contexto dos jogos, o emprego de objetivos, regras, interação, narrativa, recompensas e feedback visa proporcionar diversão e prazer, mantendo a atenção e concentração dos jogadores nos desafios propostos [Prensky 2001, Annetta et al. 2009]. Jogos sérios mesclam o entretenimento com o aprendizado, com proposta direcionada às áreas de Educação [Girard et al. 2013, Fitchat and Jordaan 2016], de modo que o equilíbrio presente no fluxo (Flow) $)^{1}$ oportunize estabilidade entre os desafios do jogo e as habilidades do aluno [Hamari et al. 2016] e, aliado às recompensas presentes e outros fatores inerentes dos jogos, é exequível aumentar a motivação dos alunos. A associação entre gráficos e sons torna a atividade mais agradável e ativa [Annetta et al. 2009] fazendo com que o aluno permaneça por mais tempo estudando e recebendo feedbacks visíveis e imediatos, o que reduz a distância entre o esforço e o sucesso. Deste modo, infere-se que o emprego de jogos pode promover maior predisposição de aprender por parte dos alunos, condição essencial de acordo com a fundamentação teórica adotada.

\subsection{Trabalhos Relacionados}

Nesta seção, são listados alguns trabalhos encontrados no Portal de Periódicos CAPES, IEEE Xplore, Elsevier, anais do Workshop sobre Educação em Computação (WEI) e pelo mecanismo de busca Google scholar, publicados entre 2011 e 2020 que descrevem a utilização ou criação de jogos digitais como meio de ensino e aprendizagem de algoritmos. Ao final é feita uma síntese de algumas características identificadas a respeito do desenvolvimento e utilização de jogos neste contexto.

O artigo de [de Oliveira et al. 2014] apresenta um experimento de um projeto de extensão para ensinar conceitos de Computação a alunos do $9^{\circ}$ ano do Ensino Fundamental. Utilizando o Scratch, os autores verificaram que todos os participantes alegaram interesse em usar a ferramenta e a maioria classificou a aula como excelente. De modo similar [Dantas et al. 2019] utilizaram o jogo Robotizen para ensinar Lógica de Programação no Ensino Fundamental. As observações mostram maior interesse dos participantes e pouca dificuldade em aplicar os conceitos de algoritmos.

Os jogos Game Logic [Netto et al. 2017] e Takkou [Barbosa et al. 2011] são exemplos que utilizam programação em blocos para ensinar Lógica de Programação por meio de comandos que direcionam o personagem e criação de estruturas de decisão e repetição. Em avaliação preliminar, [Netto et al. 2017] citam melhorias necessárias e seus avaliadores afirmam conseguir relacionar o jogo com os conteúdos vistos em sala de aula. Já Takkou é fundamentado na Aprendizagem Significativa e seu diferencial é a possibilidade de o jogador criar os próprios desafios, aumentando seu protagonismo.

O jogo NoBug's Snack Bar [Vahldick et al. 2017] utiliza programação baseada em blocos com objetivo de ensinar conceitos de programação. Utilizando o modelo LA (Learning Analytics) busca-se ajustar a sequência de missões conforme o desempenho do aluno. Após o experimento, os autores afirmam que o ajuste dinâmico de dificuldade

\footnotetext{
${ }^{1}$ Fluxo diz respeito ao crescimento do nível de dificuldade do jogo em razão do aumento das habilidades do jogador [Hamari et al. 2016].
} 
permite uma experiência melhorada, por parte dos jogadores, mas há necessidade de aprimorar o LA para alunos com baixo desempenho no conteúdo.

O jogo AlgoGame foi projetado com o objetivo de introduzir conceitos básicos de algoritmos, buscando evidenciar melhorias no desempenho dos alunos [Debabi and Bensebaa 2016]. Aperfeiçoamentos viabilizaram a seleção de fases e conteúdos apropriados conforme as necessidades do aluno [Debabi and Champagnat 2017].

Trabalhos realizados por diversas autores buscam verificar a eficiência do uso de jogos sérios para o ensino de programação, destacando fatores como poucos estudos no nível de graduação; desconsideração de competências e conhecimentos prévios do usuário; falta de diversão como fator de engajamento; pouco feedback imediato na presença de erro [Shahid et al. 2019]; carência de avaliação do jogo por especialistas; dificuldade no acesso dos jogos [Miljanovic and Bradbury 2018]; falta de análise suficientemente rigorosa [da Silva et al. 2014]; e existência de poucos processos para o desenvolvimento de jogos educacionais amplamente aceitos [Battistella et al. 2014].

Embora considerações importantes quanto a avaliação, [da Silva et al. 2014] relatam que 28 dos 29 estudos analisados apresentam melhorias no aprendizado dos participantes. De modo similar, ao avaliar jogos para o auxílio de aprendizagem de Engenharia de Software, publicados nos anais do WEI (Workshop sobre Educação em Computação) entre 2008 e 2015, [Souza and França 2016] mostram que, dos 20 trabalhos selecionados, 9 afirmaram aumento de autoconfiança técnica dos alunos; em 19 a prática não provocou sobrecarga cognitiva; 10 afirmam que o aluno percebe a utilidade do que aprendeu; e 18 permitem explorar a criatividade. Uma vez verificado o potencial da utilização de jogos e visando contribuir com as pesquisas nessa temática, o presente trabalho procurou resolver as principais lacunas, apontadas no parágrafo anterior, desenvolvendo um enredo criativo, propondo atividades teoricamente fundamentadas, e oferecendo jogabilidade e funcionalidades que promovam maior predisposição, por parte do aluno, a continuar aprendendo conteúdos de algoritmos enquanto joga.

\section{Metodologia}

O projeto maior ao qual este trabalho se vincula envolve uma pesquisa explicativa de caráter quali-quantitativo com aprovação ética (CAEE $n^{\circ}$ 40591920.4.0000.0107). As etapas de desenvolvimento são baseados na Design Based-Research que apresenta característica intervencionista buscando uma influência real na Educação e validar conceitos teóricos possibilitando compreender, avaliar e reprojetar [Anderson and Shattuck 2012]. O processo constitui-se de ciclos iterativos de prototipagem e testes, gerando contributos teóricos e práticos. Unity 3D (unity.com/pt) é usada no desenvolvimento dos jogos e PHP na construção do espaço que integra todos os recursos do ambiente online.

Para o presente trabalho, optou-se por relatar a primeira avaliação realizada a partir da liberação de algumas funcionalidades do ambiente online desenvolvido. Uma vez que foi constatada pouca existência de trabalhos na graduação, conduziu-se um estudo de caso com uma turma de 21 alunos matriculados na disciplina de Algoritmos do curso de Ciência da Computação da Universidade Estadual do Oeste do Paraná (UNIOESTE), sendo que 14 dos 17 que responderam a pesquisa inicial já reprovaram na disciplina anteriormente. Embora não seja o principal foco desse trabalho, a sequência didática foi 
desenvolvida em conjunto com a docente responsável pela disciplina de Algoritmos, em conformidade com os referenciais de formação em Computação [Zorzo et al. 2017], da Sociedade Brasileira de Computação (SBC), e o currículo de referência em tecnologia e computação [Raabe et al. 2018], refletindo na disposição dos desafios presentes nos jogos desenvolvidos. O recorte preliminar, que compreende os 3 primeiros jogos, visa identificar melhorias necessárias ao ambiente online e seu potencial de aprendizagem.

Para tal foram propostos dois questionários online no Google Forms: um aplicado no início das aulas (forms.gle/Lh8ZV6QBRBxUiYbE7) e outro 3 semanas após a disponibilização do ambiente (forms.gle/HEDykEXEFiUhEczN6). Para esse segundo questionário incorporou-se parte do instrumento de [Santos and Alves 2018], cujo objetivo é avaliar o potencial de aprendizagem da ferramenta, baseado no LORI (Learning Object Review Instrument) e GameFlow, metodologias de avaliação destinadas a analisar os objetos de aprendizagem e satisfação em jogar [Oliveira et al. 2015]. O acesso ao ambiente ficou disponível pela Internet durante toda a pesquisa, possibilitando a realização das atividades por meio de um computador e a qualquer momento. Ademais houve duas ocasiões durante o tempo de aula, que estão sendo realizadas de modo remoto e síncrono, destinados exclusivamente às atividades envolvendo o uso do ambiente.

$\mathrm{O}$ ambiente online constitui-se de um sistema hospedado em um servidor da universidade (inf.unioeste.br/ daniel/). Em síntese, seu objetivo é ser um ambiente Potencialmente Significativo que acompanhe o desenvolvimento do estudante na aprendizagem dos conteúdos, gerenciando o acesso aos jogos - de acordo com o desempenho do estudante - e apresentando materiais visuais e vídeos que explicam o enredo. O Unity $3 D$ foi utilizado para o desenvolvimento dos jogos incorporados ao ambiente. Para tornar as atividades mais dinâmicas e individualizadas, os objetos e desafios de cada fase do jogo são carregados de um banco de dados $M y S Q L$, considerando o desempenho atual de cada estudante. Essa arquitetura possibilita grande adaptabilidade aos desenvolvedores permitindo, sem alterar o código fonte, criar novas fases; gerir recompensas; adicionar informações didáticas; criar novos diálogos; entre outros fatores.

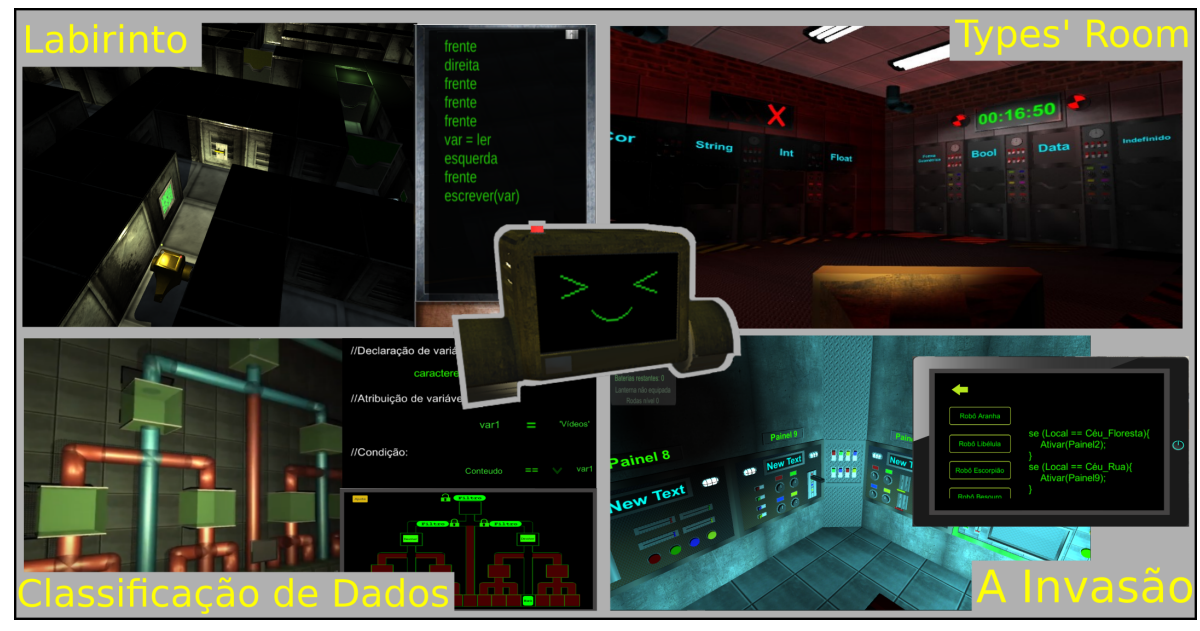

Figura 1. Os quatro primeiros jogos do ambiente

Os jogos propostos (Figura 1) são ambientados em cenários tridimensionais e interligados pelo enredo, de modo que cada jogo seja específico para ensinar alguns obje- 
tivos instrucionais e a narrativa procure despertar a motivação em continuar jogando. Ao interagir com o ambiente pela primeira vez, o aluno acessa um vídeo (https://youtu.be/dPmzsI1oO8) que apresenta o enredo do jogo. O enredo busca propiciar a reflexão sobre uma entidade artificial (Gaya) que chegou à conclusão de que deve destruir a espécie humana para salvar o planeta. Além de apresentar conceitos teóricos e práticos da Computação, discute-se sobre a preservação do ambiente e os possíveis papéis da Inteligência Artificial. Logo após assistir ao vídeo, um menu de ajuda contém informações sobre as funcionalidades do ambiente e diversas funcionalidades podem ser utilizadas (Figura 2): o mapa para acessar os jogos; informações sobre habilidades do usuário; o caderno com recortes de jornais, dicas e conteúdos teóricos, mostrados conforme se avança nos jogos; e o inventário para evoluir o personagem.

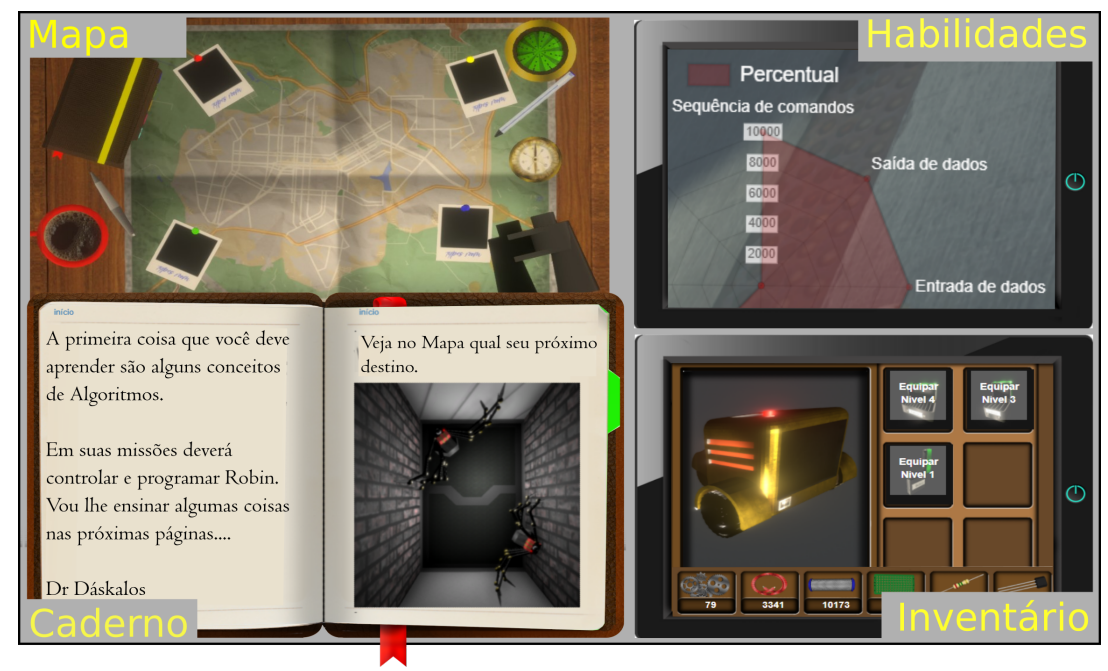

Figura 2. Principais funcionalidades do ambiente

Todos os jogos apresentam feedback visuais ou sonoros na presença de erros e, em situações específicas, são exibidas informações para auxiliar o aluno. Para aumentar a imersão no enredo, as recompensas viabilizam comprar e evoluir itens, possibilitando obter melhor iluminação, maior velocidade, entre outros. No primeiro jogo, Labirinto, o aluno deve criar algoritmos para movimentar o personagem por entre labirintos utilizando, a princípio, programação visual e depois devendo escrever cada comando. Conforme avança, são apresentados comandos de saída de dados (escrever em painéis de acesso) e de entrada de dados (escanear $Q R$ codes). Os labirintos podem ter mais de um caminho possível e o aluno deve procurar aquele que minimiza linhas de código. Do contrário, é apresentado o número de comandos necessário para concluir a mesma fase por um caminho alternativo. Ao alcançar experiência suficiente nos objetivos instrucionais de um jogo, o aluno assiste a um vídeo que mostra a continuação da história: trata-se de um vídeo interativo, que viabiliza dialogar com o personagem antagonista da história, Gaya. Feito isso, um novo jogo é liberado.

O segundo jogo, Types' Room, é usado para ensinar, em um nível conceitual, os tipos de dados. O aluno deve controlar um robô para colocar, dentro de um limite de tempo, diversos blocos em locais específicos, de acordo com o tipo de dado. Para considerar experiência prévias do jogador, de início as informações são sobre objetos do cotidiano, como formas geométricas e cores, e aos poucos são apresentados os tipos 
primitivos de dados, inteiros, reais, lógicos e caractere.

O terceiro jogo, Classificação de Dados, viabiliza a prática da declaração e atribuição de dados à variáveis e construção de estruturas condicionais. No cenário, uma rede de encanamentos guia diversos blocos com informações por caminhos diferentes. Ao final de cada caminho há um baú e, verificando o tipo de informação que deve chegar em cada um destes, o aluno deve programar os filtros entre os encanamentos, criando estruturas condicionais para conduzir os blocos pelo caminho correto. Ao final, um pseudocódigo é apresentado referente a todos os filtros configurados. Assim, pretende-se viabilizar que o aluno crie condições simples e aninhadas, trabalhando em paralelo os conhecimentos prévios tipos de dados e atribuição de variáveis.

No quarto jogo, A Invasão, o enredo mostra a antagonista Gaya atacando o local em que o protagonista mora, forçando-o a defendê-lo utilizando um banker. O aluno deve ficar atento às quatro câmeras de segurança e, ao avistar um inimigo, deve ativar um dos 12 painéis da sala, utilizando diversos elementos interativos. Para saber qual painel ativar e qual é a combinação correta, o aluno deve interpretar um código com diversas condicionais em níveis crescentes de dificuldade: condições simples (do tipo se, senão; e do tipo se, senão se, e senão); condições aninhadas; condições com conjunção (e); e condições com disjunção $(\mathrm{ou})$. Resgatando o entendimento dos conhecimentos prévios, pretende-se oportunizar a compreensão de códigos com diversas estruturas de condição. $\mathrm{O}$ quinto jogo, ainda em desenvolvimento, está situado em uma antiga universidade e contará com a resolução de desafios envolvendo, principalmente, estruturas de repetição.

\section{Resultados}

Analisando as 17 respostas obtidas no questionário inicial, foi possível verificar algumas características da turma. Quanto aos motivos para escolherem o curso (múltipla escolha) houve 13 afirmações que se referiam ao gosto por internet e jogos, 10 ao emprego futuro e 9 porque acreditam que é adequado ao seu perfil. Grande parte dos alunos acha a disciplina de Algoritmos difícil (8 razoavelmente e 7 bastante difícil) e as maiores dificuldades estão na estruturação do programa e em entender o enunciado dos exercícios. Os principais motivos que contribuíram para as reprovações foram: trabalhos difíceis e extensos; falta de estudo; dificuldade para entender as explicações; falta de frequência às aulas e alguns comentários incluíram a falta de conhecimento prévio na área, por parte dos ingressantes. Por fim, 13 afirmam jogar jogos digitais com alta frequência, mas a maioria (11) afirma nunca jogar ou jogar poucas vezes jogos educacionais.

Após a aplicação do segundo questionário percebeu-se que o uso do ambiente pode ter estimulado a motivação dos participantes. Isso porque, em uma escala likert (0-5) obteve-se uma média e desvio-padrão de 3,1 $\pm 1,2$ em relação à motivação em continuar aprendendo algoritmos e de 3,5 $\pm 0,9$ no sentimento de engajamento ao utilizar o ambiente. Quanto a acreditar que o ambiente auxiliou na compreensão dos conteúdos, obteve-se uma média e desvio-padrão de 3,1 $\pm 1,0$. Já ao serem questionados sobre a influência do ambiente no desempenho e aprendizagem, 62,5\% (10) afirmaram influência positiva - justificada pelos respondentes pela interatividade, prática dos conteúdos, fácil visualização e maior diversão - enquanto os 37,5\% (6) restantes não souberam dizer.

Quanto às melhorias sugeridas, várias sugestões foram propostas no sentido de tornar as atividades mais rápidas, diminuir o número de fases e correção de alguns er- 
ros pontuais. Essas sugestões estavam em conformidade com o tempo de dedicação dos alunos, uma vez que 56,3\% afirmam utilizar o ambiente de 1 a 3 horas por semana e os $43,7 \%$ restante menos que 1 hora. Os acessos ao ambiente confirmam isso, visto que boa parte dos estudantes só o acessou durante as atividades em horário de aula e a maioria conseguiu somente concluir todas as fases do primeiro jogo. Muitos $(83,3 \%$ das respostas) afirmaram não ter tempo para acessar os jogos fora do horário da aula. Já no que diz respeito à dificuldade em entender e resolver os desafios, obteve-se média e desvio-padrão de $1,1 \pm 1,1$ e $1,3 \pm 1,1$, respectivamente, na escala likert (0-5).

A última parte do questionário, baseada em [Santos and Alves 2018], procurou verificar o potencial de aprendizagem do ambiente. Foi utilizada uma escala likert (04) e obteve-se os seguintes resultados: média e desvio-padrão de 2,9 $\pm 1,1$ no que diz respeito ao feedback imediato e construtivo; 3,4 $\pm 0,7$ quanto aos objetivos serem claros; 3,2 \pm 1 na avaliação do enredo/narrativa; $2,9375 \pm 0,8$ quanto a interatividade; 3,6 $\pm 0,7$ para a integração de conceitos; 3,4 $\pm 0,8$ para a curva de aprendizado equilibrada; e 3,2 \pm 1 quanto a apresentação de níveis de dificuldade crescentes. Ponderando os resultados conforme definido por [Santos and Alves 2018], desconsiderando situações que exijam prática colaborativa, uma vez que não foi disponibilizado tal recurso no ambiente por não fazer parte do escopo do projeto, o ambiente apresenta uma nota de 81 (entre 0 e 100). No que tange às práticas colaborativas considerou-se que a interação entre alunos, professor e pesquisadores é possível durante tempo de aula e por outros meios, como $e$ mail, plataforma Teams, chat, além de atividades em grupo da disciplina. O emprego do ambiente procurou centrar-se no desempenho individual dos alunos.

\section{Considerações finais, trabalhos em andamento e futuros}

A avaliação mostrou, dentre outros aspectos, que o ambiente online em desenvolvimento apresenta alto potencial para aprendizagem e, de modo geral, viabiliza maior motivação aos alunos, podendo contribuir em uma das questões fundamentais da Teoria da Aprendizagem Significativa: a predisposição em aprender. A existência de feedback em cada erro cometido (mais presente no $2^{\circ}$ jogo), a retomada de conhecimentos anteriores e a característica dinâmica de oferecer mais atividades similares aos alunos com desempenho menor, parecem oferecer uma sequência didática equilibrada e mais personalizada, o que caracteriza um ponto bastante positivo conforme a fundamentação teórica adotada. Houve indicações positivas também quanto ao enredo e a interface do ambiente. As observações decorrentes desta primeira avaliação reforçaram decisões teóricas, técnicas e de projeto quanto à sua concepção indicando que tais opções estão sendo conduzidas de maneira apropriada e confrontando limitações encontradas na literatura.

Uma limitação quanto aos resultados obtidos se deve ao tamanho da amostra, pois foi realizada com uma turma com poucos alunos matriculados. Embora este aspecto, considerando a atual fase de desenvolvimento e os objetivos traçados para esta primeira avaliação, entendeu-se que esses resultados preliminares foram relevantes e reforçaram os encaminhamentos para a continuidade na condução das atividades previstas.

Quanto aos trabalhos em andamento, está sendo finalizado o desenvolvimento do quinto jogo e também do enredo. Serão retomados conteúdos anteriores e abordadas estruturas de repetição, sendo que cada desafio no cenário será avaliado e receberá um $f e$ edback específico. Ademais será realizada uma entrevista e analisados dados relativos ao 
desempenho individual de cada participante do presente experimento, que já cursou a disciplina em anos anteriores. Quanto aos trabalhos futuros, estão previstos outros momentos de avaliação, ampliando o número de participantes (portanto, da amostra), e pretende-se fazer uma avaliação com especialistas da Computação objetivando verificar o potencial educacional e abrangência dos conteúdos. Com tais dados será feita uma triangulação para verificar se o ambiente é Potencialmente Significativo. Após todas as correções necessárias o ambiente online será disponibilizado ao público, possibilitando que qualquer pessoa e instituições de ensino o utilizem livremente por meio da Internet.

\section{Referências}

Anderson, T. and Shattuck, J. (2012). Design-based research: A decade of progress in education research? Educational researcher, 41(1):16-25.

Annetta, L. A., Minogue, J., Holmes, S. Y., and Cheng, M.-T. (2009). Investigating the impact of video games on high school students' engagement and learning about genetics. Computers \& Education, 53(1):74-85.

Ausubel, D. P. (2000). Aquisição e retenção de conhecimentos: uma perspectiva cognitiva, volume 1. Lisboa: Plátano.

Barbosa, L. S., Fernandes, T. C., and Campos, A. M. (2011). Takkou: Uma ferramenta proposta ao ensino de algoritmos. In XVIII Workshop sobre Educação em Computação.

Battistella, P., von Wangenheim, C., and Fernandes, J. M. (2014). Como jogos educacionais são desenvolvidos? uma revisão sistemática da literatura. In Anais do XXII Workshop sobre Educação em Computação, pages 159-168. SBC.

da Silva, T. R., Medeiros, T. J., and Aranha, E. H. d. S. (2014). Jogos digitais para ensino e aprendizagem de programação: uma revisão sistemática da literatura. In Brazilian Symposium on Computers in Education (SBIE), volume 25, page 692.

Dantas, I., Neto, J., Silva, L., Neto, L., Lima, D., Scaico, P., and Costa, T. (2019). Ensino de lógica de programação no ensino fundamental utilizando o jogo robotizen: um relato de experiência. In Anais do XXVII Workshop sobre Educação em Computação, pages 51-60. SBC.

de Oliveira, M., de Souza, A., Ferreira, A., and Barreiros, E. (2014). Ensino de lógica de programação no ensino fundamental utilizando o scratch: um relato de experiência. In Anais do XXII Workshop sobre Educação em Computação, pages 239-248. SBC.

Debabi, W. and Bensebaa, T. (2016). Using serious game to enhance learning and teaching algorithmic. Journal of e-Learning and Knowledge Society, 12(2).

Debabi, W. and Champagnat, R. (2017). Towards architecture for pedagogical and game scenarios adaptation in serious games. International Association for Development of the Information Society.

Fitchat, L. and Jordaan, D. (2016). Ten heuristics to evaluate the user experience of serious games. International Journal of Social Sciences and Humanity Studies, 2(8):209-225.

Giraffa, M. M. and da Costa Mora, M. (2016). Evasão na disciplina de algoritmo e programação: um estudo a partir dos fatores intervenientes na perspectiva do aluno. In Congresos CLABES. 
Girard, C., Ecalle, J., and Magnan, A. (2013). Serious games as new educational tools: how effective are they? a meta-analysis of recent studies. Journal of Computer Assisted Learning, 29(3):207-219.

Hamari, J., Shernoff, D. J., Rowe, E., Coller, B., Asbell-Clarke, J., and Edwards, T. (2016). Challenging games help students learn: An empirical study on engagement, flow and immersion in game-based learning. Computers in human behavior, 54:170179.

Hodges, C., Moore, S., Lockee, B., Trust, T., and Bond, A. (2020). The difference between emergency remote teaching and online learning. Educause Review, 27.

Miljanovic, M. A. and Bradbury, J. S. (2018). A review of serious games for programming. In Joint International Conference on Serious Games, pages 204-216. Springer.

Moreira, M. A. (2010). O que é afinal aprendizagem significativa? 2010. Aula Inaugural do Programa de Pós-Graduação em Ensino de Ciências Naturais, Instituto de Física, Universidade Federal do Mato Grosso, Cuiabá, MT, 23.

Netto, D., Medeiros, L. M., de Pontes, D., and de Morais, E. (2017). Game logic: Um jogo para auxiliar na aprendizagem de lógica de programação. In Anais do XXV Workshop sobre Educação em Computação. SBC.

Oliveira, W., Neto, S., da Silva Junior, C. G., and Bittencourt, I. I. (2015). Avaliação de jogos educativos: Uma abordagem no ensino de matemática. In Brazilian Symposium on Computers in Education (SBIE), page 657.

Petrovskaya, E. (2019). Not all fun and games: The design and evaluation of a game to increase intrinsic motivation in learning programming. University College London.

Prensky, M. (2001). Digital Game-based Learning. McGraw-Hill.

Raabe, A. L. A., Brackmann, C. P., and Campos, F. R. (2018). Currículo de referência em tecnologia e computação: da educação infantil ao ensino fundamental. Centro de Inovação para a Educação Básica-CIEB. Disponível em: curriculo.cieb.net.br.

Santos, W. and Alves, L. (2018). Uma taxonomia avaliativa para jogos digitais educacionais. In Proceedings of SBGames 2018.

Shahid, M., Wajid, A., Haq, K. U., Saleem, I., and Shujja, A. H. (2019). A review of gamification for learning programming fundamental. In 2019 International Conference on Innovative Computing (ICIC), pages 1-8. IEEE.

Souza, M. and França, C. (2016). O que explica o sucesso de jogos no ensino de engenharia de software? uma teoria de motivação. In Anais do XXIV Workshop sobre Educação em Computação, pages 260-269. SBC.

Vahldick, A., Mendes, A. J., and Marcelino, M. J. (2017). Dynamic difficulty adjustment through a learning analytics model in a casual serious game for computer programming learning. EAI Endorsed Transactions on Serious Games, 4(13).

Zorzo, A. F., Nunes, D. J., Matos, E. d. S., Steinmacher, I. F., Leite, J. C., De Araujo, R. M., Correia, R. C. M., and Martins, S. e. L. (2017). Referenciais de formação para os cursos de graduação em computação. 